\title{
Gel Forming Drop Solution Dosage Form
}

National Cancer Institute

\section{Source}

National Cancer Institute. Gel Forming Drop Solution Dosage Form. NCI Thesaurus.

Code C60994.

A solution intended for administration in a drop-wise fashion; it becomes gelatinous upon administration. 